International Journal of Pure and Applied Mathematical Sciences.

ISSN 0972-9828 Volume 9, Number 2 (2016), pp. 123-131

(C) Research India Publications

https://dx.doi.org/10.37622/IJPAMS/9.2.2016.123-131

\title{
CONGRUUM PROBLEM
}

\author{
Manju Somanath ${ }^{1}$ and J. Kannan ${ }^{2}$ \\ ${ }^{1}$ Assistant Professor, Department of Mathematics, \\ National College, Trichy - 01, India \\ ${ }^{2}$ Research Scholar, Department of Mathematics, \\ National College, Trichy - 01, India
}

\begin{abstract}
The ternary quadratic Diophantine equation $X^{2}+Z^{2}=2 Y^{2}$ is considered and a few interesting properties among the solutions are presented.

Keywords - Arithmetic progression, Diophantine equation, Integral solution, Congruum.
\end{abstract}

\section{NOTATIONS USED:}

(i) $\quad P(n)=n(n+1)$ Pronic number of rank $n$.

(ii) $S_{n}=6 n^{2}-6 n+1$ Star number of rank $n$.

(iii) $\quad \mathbf{N}^{*}=\{0\} \cup \mathbf{N}$ Non negative integers.

\section{INTRODUCTION}

Number theory is the branch of Mathematics concerned with studying the properties and relations of integers. In Number Theory, a congruum (plural congrua) is the difference between successive square numbers in an arithmetic progression of three squares equally spaced apart from each other. Then the space between them, $Z^{2}-$ $Y^{2}=Y^{2}-X^{2}$ is called a congruum.

For instance, the number 96 is a congruum since it is the difference between each pair of three squares 4,100 \& 196. The first few congrua are 24, 96, 120, 216, 240, 
$336,384,480, \ldots$ If $X, Y, Z$ represents the sides of the Pythagorean triangle then the congruum is itself four times the area of the Pythagorean triangle.

The two right triangles with leg and hypotenuse $(7,13)$ and $(13,17)$ have equal third sides of length $\sqrt{ } 120$. The square of this side, 120, is a congruum: it is the difference between consecutive values in the Arithmetic progression of squares $7^{2}, 13^{2}, 17^{2}$.

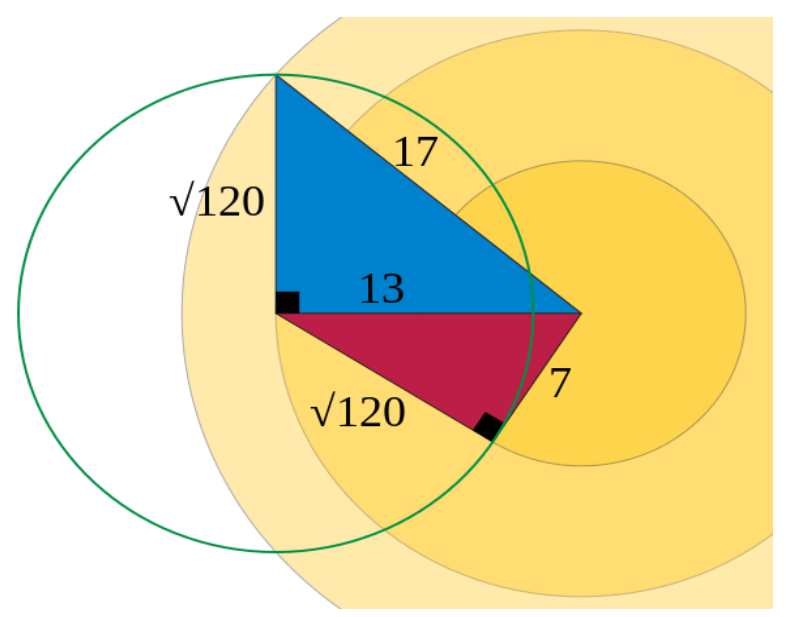

Figure 1: A geometrical example of a Congruum

Many mathematicians of the ancient years Fibonacci, Fermat, Oystein Ore etc $[1,4$, 6] studied the congruum problem. This problem can be formalized as a Diophantine equation $X^{2}+Z^{2}=2 Y^{2}$.

Diophantine problems are important because of their rich variety. In this communication, the ternary quadratic Diophantine equation $X^{2}+Z^{2}=2 Y^{2}$ is analyzed for various patterns of integer solutions.

\section{METHOD OF ANALYSIS}

In congruum problem, we want to find a number $h$ such that $Y^{2}+h=X^{2}$ and $Y^{2}-h=Z^{2}$ which gives $2 h=X^{2}-Z^{2}=(X+Z)(X-Z)$. since left hand side is even, $X-Z$ must be both odd or both even. Therefore, $X-Z$ is even, $X-Z=2 k$. Hence $X+Z=\frac{h}{k}$. Therefore $X=\frac{h}{2 k}+k$ and $Z=\frac{h}{2 k}-k$.which can be written as $Y^{2}=\left(\frac{h}{2 k}\right)^{2}+k^{2}$.

i.e., $\left(\quad Y, \frac{h}{2 k}, k\right) \quad$ forms a Pythagorean triangle with sides $t\left(m^{2}+n^{2}\right), 2 m n t, t\left(m^{2}-n^{2}\right)$. 
In this communication, the ternary quadratic Diophantine equation $X^{2}+Z^{2}=2 Y^{2}$ is analyzed for various patterns of integer solutions.

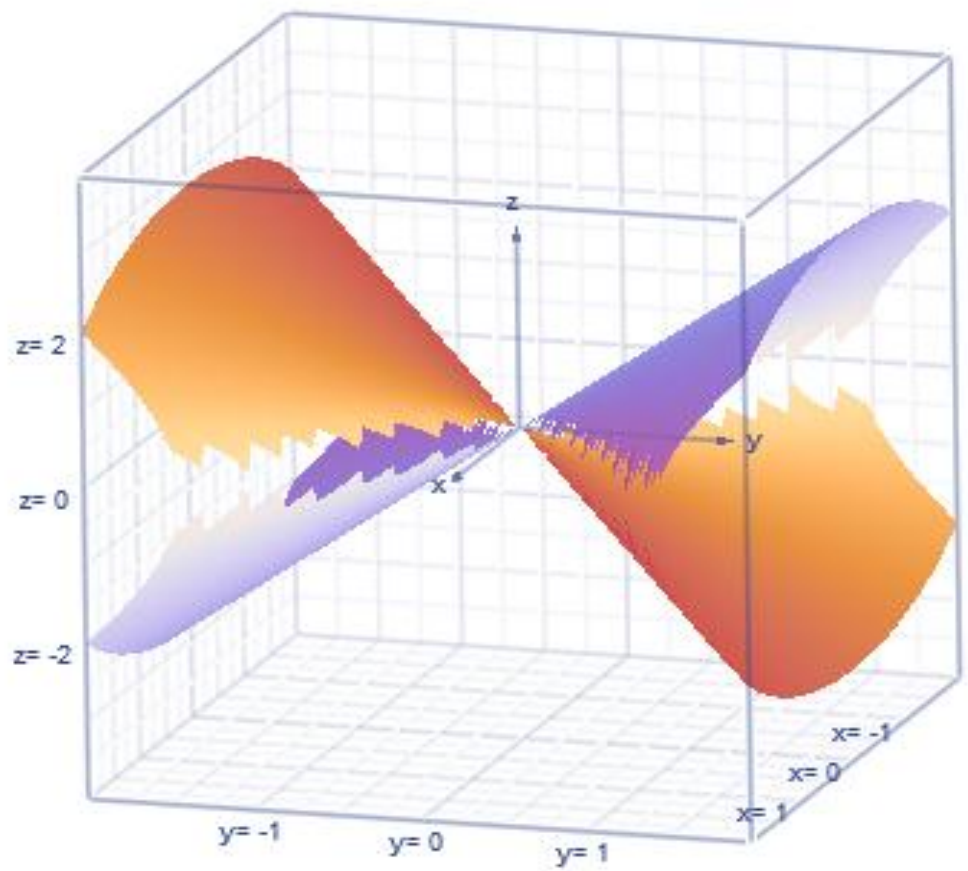

Figure 2: Pictorial representation of the equation

Considering the congruum problem as a ternary quadratic Diophantine equation with 3 unknown

$$
X^{2}+Z^{2}=2 Y^{2}
$$

We present below different patterns of integral solution of (1):

\section{A. Pattern}

The linear transformation

$$
X=u+v, Z=u-v \text { in (1) }
$$

leads to the Pythagorean equation

$$
u^{2}+v^{2}=Y^{2}
$$

with solutions

$$
u=p^{2}-q^{2}
$$




$$
\begin{aligned}
& v=2 p q \\
& Y=p^{2}+q^{2}
\end{aligned}
$$

Thus the solutions of (1) are

$$
\begin{aligned}
& X=p^{2}+2 p q-q^{2} \\
& Y=p^{2}+q^{2} \\
& Z=p^{2}-2 p q-q^{2}
\end{aligned}
$$

Some interesting properties of the above solutions are

(i) If $p=q$, then $X-Z$ is a perfect square.

(ii) $3[Y(1, n)-X(1, n)]+1$ is Star number of rank $n$.

(iii) $\frac{X(n, 1)+Y(n, 1)}{2}$ is a Pronic number of rank $n$.

(iv) If $p=q$, then $6(X-Z)$ is a Congruum.

(v) $\quad 3(X+Y+Z)$ is a Nasty number, when $q=p$.

\section{B. Pattern}

(1) can be rewritten as

$$
\begin{aligned}
X^{2}-Y^{2} & =Y^{2}-Z^{2} \\
\frac{X-Y}{Y-Z} & =\frac{Y+Z}{X+Y}=\frac{p}{q} \text { (say) }
\end{aligned}
$$

Expressing this as a system of simultaneous equations:

$$
\begin{aligned}
& q X-(p+q) Y+p Z=0 \\
& p X+(p-q) Y-q Z=0
\end{aligned}
$$

(4) and (5) solving we get

$$
\begin{gathered}
X=-p^{2}+q^{2}+2 p q \\
Y=p^{2}+q^{2} \\
Z=p^{2}-q^{2}+2 p q
\end{gathered}
$$


Choice 1:

(3) can be also written as

$$
\frac{X-Y}{Y+Z}=\frac{Y-Z}{X+Y}=\frac{p}{q}(\text { say })
$$

we obtain the solution of (1) as

$$
\begin{gathered}
X=p^{2}-q^{2}-2 p q \\
Y=-p^{2}-q^{2} \\
Z=p^{2}-q^{2}+2 p q
\end{gathered}
$$

Choice 2:

(3) can be also written as

$$
\frac{X+Y}{Y-Z}=\frac{Y+Z}{X-Y}=\frac{p}{q}(\text { say })
$$

we obtain the solution of (1) as

$$
\begin{aligned}
& X=p^{2}-q^{2}+2 p q \\
& Y=p^{2}+q^{2} \\
& Z=p^{2}-q^{2}-2 p q
\end{aligned}
$$

\section{Pattern}

The substitution

$$
\begin{aligned}
& X=u+v, \quad Y=u-v \quad \text { in (1) } \\
& u=3 v \pm \sqrt{\left(8 v^{2}+Z^{2}\right)}
\end{aligned}
$$

gives

Assume

$$
\alpha^{2}=8 v^{2}+Z^{2}
$$

Case (I)

Solving (6), we get

$$
\begin{gathered}
v=2 p q \\
Z=8 p^{2}-q^{2} \\
\alpha=8 p^{2}+q^{2}
\end{gathered}
$$


For the corresponding two values of $u$, we get the solutions of (1) as

$$
\begin{gathered}
X=8 p q+8 p^{2}+q^{2} \\
Y=4 p q+8 p^{2}+q^{2} \\
Z=8 p^{2}-q^{2}
\end{gathered}
$$

and

$$
\begin{gathered}
X=8 p q-8 p^{2}-q^{2} \\
Y=4 p q-8 p^{2}-q^{2} \\
Z=8 p^{2}-q^{2}
\end{gathered}
$$

Case (II)

Proceeding as in the above case for the choice

$$
\begin{gathered}
v=2 p q \\
Z=p^{2}-8 q^{2} \\
\alpha=p^{2}+8 q^{2}
\end{gathered}
$$

The corresponding solutions of (1) are

$$
\begin{gathered}
X=8 p q+p^{2}+8 q^{2} \\
Y=4 p q+p^{2}+8 q^{2} \\
Z=p^{2}-8 q^{2}
\end{gathered}
$$

and

$$
\begin{gathered}
X=8 p q-p^{2}-8 q^{2} \\
Y=4 p q-p^{2}-8 q^{2} \\
Z=p^{2}-8 q^{2}
\end{gathered}
$$

Case (III)

Letting $Z=1$, in (6) we get

$$
\alpha^{2}=8 v^{2}+1
$$


Solving (7) as a Pell's equation, we get

$$
u_{n}=3 v_{n} \pm \alpha_{n}
$$

where

$$
\begin{aligned}
& \alpha_{n}=\frac{1}{2}\left[(3+\sqrt{8})^{n+1}+(3-\sqrt{8})^{n+1}\right] \\
& v_{n}=\frac{1}{2 \sqrt{8}}\left[(3+\sqrt{8})^{n+1}-(3-\sqrt{8})^{n+1}\right]
\end{aligned}
$$

Sub case I

For the choice of

$$
u_{n}=\frac{3}{2 \sqrt{8}}\left[(3+\sqrt{8})^{n+1}-(3-\sqrt{8})^{n+1}\right]+\frac{1}{2}\left[(3+\sqrt{8})^{n+1}+(3-\sqrt{8})^{n+1}\right]
$$

We have the sequence of solutions of (1) as

$$
\begin{aligned}
& X_{n}=\frac{1}{2 \sqrt{8}}\left[(4+\sqrt{8})(3+\sqrt{8})^{n+1}-(4-\sqrt{8})(3-\sqrt{8})^{n+1}\right] \\
& Y_{n}=\frac{1}{2 \sqrt{8}}\left[(2+\sqrt{8})(3+\sqrt{8})^{n+1}-(2-\sqrt{8})(3-\sqrt{8})^{n+1}\right]
\end{aligned}
$$

A few numerical examples are presented in the following table:

\begin{tabular}{|c|c|c|}
\hline $\boldsymbol{X}$ & $\boldsymbol{h}$ & $\begin{array}{c}\boldsymbol{h}=\boldsymbol{X}^{\mathbf{2}}-\boldsymbol{Y}^{\mathbf{2}} \\
=\boldsymbol{Y}^{\mathbf{2}}-\boldsymbol{Z}^{\mathbf{2}}\end{array}$ \\
\hline $\mathrm{X}_{0}=7$ & $\mathrm{Y}_{0}=5$ & 24 \\
\hline $\mathrm{X}_{1}=41$ & $\mathrm{Y}_{1}=29$ & 840 \\
\hline $\mathrm{X}_{2}=239$ & $\mathrm{Y}_{2}=169$ & 28560 \\
\hline $\mathrm{X}_{3}=1393$ & $\mathrm{Y}_{3}=985$ & 970224 \\
\hline $\mathrm{X}_{4}=8119$ & $\mathrm{Y}_{4}=5741$ & 32959080 \\
\hline $\mathrm{X}_{5}=47321$ & $\mathrm{Y}_{5}=33461$ & 1119638520 \\
\hline $\mathrm{X}_{6}=275807$ & $\mathrm{Y}_{6}=195025$ & $3.803475062 \times 10^{10}$ \\
\hline
\end{tabular}


From the above solutions we observe some interesting properties:

(i) $\quad X_{n}, Y_{n}, Z_{n}$ all are odd .

(ii) $\quad\left(X_{n}, Y_{n}\right)=1$, for all $n \in \boldsymbol{N}^{*}$.

(iii) $X_{n} Y_{n}\left(X_{n}^{2}-Y_{n}^{2}\right)$ is a area of a Pythagorean triangle.

(iv) $\quad 4\left(X_{n}^{3} Y_{n}-X_{n} Y_{n}^{3}\right)$ is a congruum.

(v) $\left(X_{n}, Y_{n}, Z_{n}\right)$ is any one of the solution then, $\left(-X_{n},-Y_{n},-Z_{n}\right),\left(X_{n}, Y_{n},-Z_{n}\right)\left(X_{n},-Y_{n}, Z_{n}\right),\left(-X_{n}, Y_{n}, Z_{n}\right),\left(X_{n},-Y_{n},-Z_{n}\right)$, $\left(-X_{n},-Y_{n}, Z_{n}\right),\left(-X_{n}, Y_{n},-Z_{n}\right)$, is also solution of above equation.

(vi) $X_{6(n+1)}-X_{6 n} \equiv 0(\bmod 10)$, for all $n \in N^{*}$.

(vii) $X_{2(n+1)}-X_{2 n} \equiv 0(\bmod 10)$, for all $n$ such that $n$ and $n+1 \neq 3 m$, for any integer $m$.

(viii) $Y_{3 n} \equiv 0(\bmod 5)$, for all $n \in \boldsymbol{N}^{*}$.

(ix) $\quad Y_{3(n+1)}-Y_{3 n} \equiv 0(\bmod 10)$, for all $n \in N^{*}$

(x) $\quad Y_{n+1}-Y_{n} \equiv 0(\bmod 10)$, for all $n$ such that $n \equiv 1(\bmod 3)$.

Sub Case (II)

Substituting these values in

$$
u_{n}=3 v_{n}-\alpha_{n}
$$

Giving the solutions of (1) as,

$$
\begin{aligned}
& X_{n}=\frac{1}{2 \sqrt{8}}\left[(4-\sqrt{8})(3+\sqrt{8})^{n+1}-(4+\sqrt{8})(3-\sqrt{8})^{n+1}\right] \\
& Y_{n}=\frac{1}{2 \sqrt{8}}\left[(2-\sqrt{8})(3+\sqrt{8})^{n+1}-(2+\sqrt{8})(3-\sqrt{8})^{n+1}\right]
\end{aligned}
$$

The solutions in the above two sub cases satisfy the following recurrence relations.

(i) $\quad X_{n+2}-6 X_{n+1}+X_{n}=0$

(ii) $\quad Y_{n+2}-6 Y_{n+1}+Y_{n}=0$

(iii) $\quad Z_{n}=1$. 


\section{OBSERVATION}

Congrua is closely related to congruent numbers which is defined as the area of a Pythagorean triangle with rational sides. Every congruent number is a congruum multiplied by a square of a rational number. The problem of three squares in A.P is also closely related to congruent numbers.

\section{CONCLUSION}

To Conclude, One may search for other patterns of solutions.

\section{REFERENCES}

[1] L.E. Dickson, History of Theory of Numbers, Vol.2, Chelsea Publishing Company, New York, 1952.

[2] Dr. Manju somanath, J. Kannan and Mr. K. Raja, "Gaussian Pythagorean Triples $X^{2}+Y^{2}=Z^{2}$ ", International Journal of Engineering Research and Management, Vol. 03 Issue 04 (April 2016).

[3] Carmichael. R. D, Theory of Numbers and Diophantine Analysis, Dover Publication Inc., New York, 1952.

[4] Niven. J, Zuckerman and Montgomery, An introduction to the theory of numbers, fifth edition, John We lay, New York 1991.

[5] M. A. Gopalan, Manju somanath and N.Vanitha, "On Space Pythagorean equation $X^{2}+Y^{2}+Z^{2}=W^{2}$, International Journal of Mathematics, Computer Science and Information Technology Vol. 1, pp129-133, JanuaryJune 2007.

[6] Oystein Ore, Number Theory and its History, Dover publication, New York.

[7] Conway J H and Guy R K, "The book of Numbers", Springer Science and Business Media, 2006. 
